PROCEEDINGS OF THE

AMERICAN MATHEMATICAL SOCIETY

Volume 132, Number 6, Pages 1787-1795

S 0002-9939(04)07411-8

Article electronically published on January 30, 2004

\title{
STABILITY OF $C^{*}$-ALGEBRAS ASSOCIATED TO GRAPHS
}

\author{
MARK TOMFORDE
}

(Communicated by David R. Larson)

\begin{abstract}
We characterize stability of graph $C^{*}$-algebras by giving five conditions equivalent to their stability. We also show that if $G$ is a graph with no sources, then $C^{*}(G)$ is stable if and only if each vertex in $G$ can be reached by an infinite number of vertices. We use this characterization to realize the stabilization of a graph $C^{*}$-algebra. Specifically, if $G$ is a graph and $\tilde{G}$ is the graph formed by adding a head to each vertex of $G$, then $C^{*}(\tilde{G})$ is the stabilization of $C^{*}(G)$; that is, $C^{*}(\tilde{G}) \cong C^{*}(G) \otimes \mathcal{K}$.
\end{abstract}

\section{INTRODUCTION}

In 1980 Cuntz and Krieger introduced a class of $C^{*}$-algebras generated by families of partial isometries satisfying relations determined by a finite matrix with entries in $\{0,1\}$. These Cuntz-Krieger algebras were initially studied because of their appearance in the study of topological Markov chains. Later it was found that they also have important parallels with certain kinds of dynamical systems (e.g., shifts of finite type).

Since their inception, Cuntz-Krieger algebras have been generalized in an extraordinary number of ways. One generalization whose study has proven particularly fruitful is the $C^{*}$-algebras associated to directed graphs. In 1982 Watatani noted that one could view the Cuntz-Krieger algebra associated to a finite matrix $A$ as the $C^{*}$-algebra associated to the finite directed graph with adjacency matrix $A$ 13]. However, these ideas were not more fully explored until the late 1990s when Kumjian, Pask, Raeburn, and Renault [11] introduced $C^{*}$-algebras associated to locally finite graphs (i.e., possibly infinite graphs in which each vertex emits and receives a finite number of edges). Not long after this it was shown in [2] that many of the same results also hold for $C^{*}$-algebras associated to row-finite graphs (i.e., possibly infinite graphs in which every vertex emits finitely many edges), and often the same techniques can be applied to prove these results. In the early 2000s $C^{*}$-algebras associated to arbitrary directed graphs were finally considered [7. Unlike the generalization from locally finite to row-finite graphs, it was found that extending results to $C^{*}$-algebras of arbitrary graphs often involved significant modifications to statements of theorems as well as the development of new techniques for their proofs.

In this paper we consider the notion of stability for $C^{*}$-algebras associated to arbitrary directed graphs. Recall that a $C^{*}$-algebra $A$ is said to be stable if $A \cong$

Received by the editors June 14, 2002 and, in revised form, March 1, 2003.

2000 Mathematics Subject Classification. Primary 46L55.

(C)2004 American Mathematical Society 
$A \otimes \mathcal{K}$, where $\mathcal{K}$ denotes the compact operators on a separable infinite-dimensional Hilbert space. Furthermore, if $A$ is a $C^{*}$-algebra, then one may form its stabilization $A \otimes \mathcal{K}$. Since $\mathcal{K} \otimes \mathcal{K} \cong \mathcal{K}$, one has that the stabilization of a $C^{*}$-algebra is stable.

If $G$ is a graph and $C^{*}(G)$ is its associated $C^{*}$-algebra, then we prove in Theorem 3.2 that the stability of $C^{*}(G)$ is equivalent to five other conditions. This theorem generalizes a result of Hjelmborg [8, Theorem 2.14] in which a characterization of stability for $C^{*}$-algebras of locally finite graphs was obtained. Both of these results make use of a nontrivial characterization of stability due to Rørdam and Hjelmborg [9. However, our proof of Theorem 3.2 will involve techniques significantly different from Hjelmborg's proof of [8, Theorem 2.14]. Furthermore, in addition to applying to $C^{*}$-algebras of arbitrary graphs, Theorem 3.2 is different from [8, Theorem 2.14] in another respect, namely that it includes a characterization in terms of the graph traces on all of $G$, rather than on a special subgraph of $G$ as in Condition (d) of [8, Theorem 2.14].

In Corollary 3.3 we show that there is a particularly nice characterization of stability for $C^{*}(G)$ when $G$ has no sources: If $G$ is a graph with no sources, then $C^{*}(G)$ is stable if and only if every vertex of $G$ can be reached by an infinite number of vertices. This gives an easily verifiable condition for determining the stability of the $C^{*}$-algebra solely in terms of the graph.

Building off this characterization, in $\$ 4$ we develop a method for realizing the stabilization of a graph algebra and we show that it is also a graph algebra. If $G$ is a graph, then we obtain a new graph $\tilde{G}$ by adding a "head"

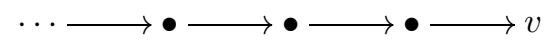

to each vertex $v$ in $G$. We prove in Theorem 4.2 that $C^{*}(\tilde{G})$ is the stabilization of $C^{*}(G)$; that is, $C^{*}(\tilde{G}) \cong C^{*}(G) \otimes \mathcal{K}$. As a corollary we have that the class of graph algebras is closed under stabilization.

\section{Preliminaries}

We provide some basic facts about graph algebras and refer the reader to [10], 2], and [1] for more details. A (directed) graph $G=\left(G^{0}, G^{1}, r, s\right)$ consists of a countable set $G^{0}$ of vertices, a countable set $G^{1}$ of edges, and maps $r, s: G^{1} \rightarrow G^{0}$ identifying the range and source of each edge. A vertex $v \in G^{0}$ is called a sink if $\left|s^{-1}(v)\right|=0$, and $v$ is called an infinite emitter if $\left|s^{-1}(v)\right|=\infty$. If $v$ is either a sink or an infinite emitter, then we call $v$ a singular vertex. A graph $G$ is said to be row-finite if it has no infinite emitters.

If $G$ is a graph, then we define a Cuntz-Krieger $G$-family to be a set of mutually orthogonal projections $\left\{p_{v}: v \in G^{0}\right\}$ and a set of partial isometries $\left\{s_{e}: e \in G^{1}\right\}$ with orthogonal ranges that satisfy the Cuntz-Krieger relations:

(1) $s_{e}^{*} s_{e}=p_{r(e)}$ for every $e \in G^{1}$;

(2) $s_{e} s_{e}^{*} \leq p_{s(e)}$ for every $e \in G^{1}$;

(3) $p_{v}=\sum_{s(e)=v} s_{e} s_{e}^{*}$ for every $v \in G^{0}$ that is not a singular vertex.

The graph algebra $C^{*}(G)$ is defined to be the $C^{*}$-algebra generated by a universal Cuntz-Krieger $G$-family.

A path in $G$ is a sequence of edges $\alpha=\alpha_{1} \alpha_{2} \ldots \alpha_{n}$ with $r\left(\alpha_{i}\right)=s\left(\alpha_{i+1}\right)$ for $1 \leq$ $i<n$, and we say that $\alpha$ has length $|\alpha|=n$. We let $G^{n}$ denote the set of all paths of length $n$, and we let $G^{*}:=\bigcup_{n=0}^{\infty} G^{n}$ denote the set of finite paths in $G$. Note that vertices are considered paths of length zero. The maps $r, s$ extend to $G^{*}$, and for 
$v, w \in G^{0}$ we write $v \geq w$ if there exists a path $\alpha \in G^{*}$ with $s(\alpha)=v$ and $r(\alpha)=w$. Also for a path $\alpha:=\alpha_{1} \ldots \alpha_{n}$ we define $s_{\alpha}:=s_{\alpha_{1}} \ldots s_{\alpha_{n}}$. It is a consequence of the Cuntz-Krieger relations that $C^{*}(G)=\overline{\operatorname{span}}\left\{s_{\alpha} s_{\beta}^{*}: \alpha, \beta \in G^{*}\right.$ and $\left.r(\alpha)=r(\beta)\right\}$.

We say that a path $\alpha:=\alpha_{1} \ldots \alpha_{n}$ of length 1 or greater is a loop if $r(\alpha)=s(\alpha)$, and we call the vertex $s(\alpha)=r(\alpha)$ the base point of the loop. A loop is said to be simple if $s\left(\alpha_{i}\right) \neq s\left(\alpha_{1}\right)$ for all $1<i \leq n$. The following is an important condition for graphs to satisfy.

Condition K. No vertex in $G$ is the base point of exactly one simple loop; that is, every vertex is either the base point of no loops or at least two simple loops.

The graph algebra $C^{*}(G)$ is unital if and only if $G$ has a finite number of vertices (cf. [10, Proposition 1.4]) and in this case $1_{C^{*}(G)}=\sum_{v \in G^{0}} p_{v}$. If $G$ has an infinite number of vertices and we list them as $G^{0}=\left\{v_{1}, v_{2}, \ldots\right\}$ and define $p_{n}:=\sum_{i=1}^{n} p_{v_{i}}$, then $\left\{p_{n}\right\}_{n=1}^{\infty}$ will be an approximate unit for $C^{*}(G)$.

Definition 2.1. A trace on a $C^{*}$-algebra $A$ is a linear functional $\tau: A \rightarrow \mathbb{C}$ with the property that $\tau(a b)=\tau(b a)$ for all $a, b \in A$. We say that $\tau$ is positive if $\tau(a) \geq 0$ for all $a \in A^{+}$. If $\tau$ is a positive trace and $\|\tau\|=1$ we call $\tau$ a tracial state. The set of all tracial states is denoted $T(A)$.

Definition 2.2. If $G$ is a graph, then a graph trace on $G$ is a function $g: G^{0} \rightarrow \mathbb{R}^{+}$ with the following two properties:

(1) For any $v \in G^{0}$ with $0<\left|s^{-1}(v)\right|<\infty$ we have $g(v)=\sum_{s(e)=v} g(r(e))$.

(2) For any infinite emitter $v \in G^{0}$ and any finite set of edges $e_{1}, \ldots, e_{n} \in$ $s^{-1}(v)$ we have $g(v) \geq \sum_{i=1}^{n} g\left(r\left(e_{i}\right)\right)$.

Because the value of $g$ at any vertex is nonnegative, it follows that whenever $v$ is an infinite emitter the infinite sum $\sum_{s(e)=v} g(r(e))$ converges, and moreover $\sum_{s(e)=v} g(r(e)) \leq g(v)$.

We define the norm of $g$ to be the (possibly infinite) value $\|g\|:=\sum_{v \in G^{0}} g(v)$. We shall call a graph trace bounded if $\|g\|<\infty$, and we shall use $T(G)$ to denote the set of all graph traces on $G$ with norm one. Also note that if $v, w \in G^{0}$, then $v \geq w$ implies $g(v) \geq g(w)$.

If $\tau: C^{*}(G) \rightarrow \mathbb{C}$ is a tracial state, then $\tau$ induces a graph trace $g_{\tau}$ of norm one given by $g_{\tau}(v):=\tau\left(p_{v}\right)$. If $G$ satisfies Condition (K), then the map $\tau \mapsto g_{\tau}$ is a bijection (in fact, an affine homeomorphism) from $T\left(C^{*}(G)\right.$ ) onto $T(G)$ [12, §3]. There are examples which show that in general this map is not injective.

Definition 2.3. We say that two projections $p, q \in A$ are equivalent, written $p \sim q$, if there exists an element $v \in A$ with $p=v v^{*}$ and $q=v^{*} v$.

In 4] Joachim Cuntz introduced a notion of comparison of (positive) elements in a $C^{*}$-algebra for the purpose of constructing dimension functions and traces on $C^{*}$-algebras.

Definition 2.4 (Cuntz). Let $A$ be a $C^{*}$-algebra, and let $a, b$ be positive elements in $A$. We write $a \lesssim b$ if there exists a sequence $\left\{x_{k}\right\}_{k=1}^{\infty}$ in $A$ with $x_{k}^{*} b x_{k} \rightarrow a$.

If $p, q$ are projections in a $C^{*}$-algebra $A$, then $p \lesssim q$ if and only if $p$ is equivalent to a subprojection of $q$; that is, there exists a partial isometry $v \in A$ such that $p=v v^{*}$ and $v^{*} v \leq q$. Thus the above definition agrees with usual definition of comparison of two projections. 
If $e \in G^{1}$, then we see that $p_{r(e)}=s_{e} s_{e}^{*}$ and $s_{e} s_{e}^{*} \leq p_{s(e)}$. Therefore, $p_{r(e)} \lesssim p_{s(e)}$. More generally, we see that $v \geq w$ implies $p_{w} \lesssim p_{v}$.

Definition 2.5. If $G$ is a graph, a subset $H \subseteq G^{0}$ is said to be hereditary if for every $e \in G^{1}$ we have that $s(e) \in H$ implies $r(e) \in H$. A hereditary subset is said to be saturated if whenever $v \in G^{0}$ with $0<\left|s^{-1}(v)\right|<\infty$, then $\{r(e): e \in$ $G^{1}$ and $\left.s(e)=v\right\} \subseteq H$ implies that $v \in H$. If $H$ is a hereditary subset, then the saturation of $H$ is the smallest saturated hereditary subset $\bar{H}$ of $G^{0}$ containing $H$.

If $H$ is a hereditary subset of $G^{0}$, then we can give an inductive description of the saturation $\bar{H}$. We define $H_{0}:=H$ and having defined $H_{n}$ we set

$$
H_{n+1}:=H_{n} \cup\left\{v \in G^{0}: 0<\left|s^{-1}(v)\right|<\infty \text { and } s(e)=v \text { implies } r(e) \in H_{n}\right\} .
$$

Then it is straightforward to show that $\bar{H}=\bigcup_{n=0}^{\infty} H_{n}$.

Definition 2.6. Given a saturated hereditary subset $H \subseteq G^{0}$, we define

$$
B_{H}:=\left\{v \in G^{0}:\left|s^{-1}(v)\right|=\infty \text { and } 0<\left|s^{-1}(v) \cap r^{-1}\left(G^{0} \backslash H\right)\right|<\infty\right\} .
$$

Since $H$ is hereditary, we see that $B_{H}$ is disjoint from $H$. If $\left\{s_{e}, p_{v}\right\}$ is a generating Cuntz-Krieger $G$-family in $C^{*}(G)$, then for $S \subseteq B_{H}$ we define

$$
I_{(H, S)}:=\text { the ideal in } C^{*}(G) \text { generated by }\left\{p_{v}: v \in H\right\} \cup\left\{p_{v}^{H}: v \in S\right\},
$$

where

$$
p_{v}^{H}:=p_{v}-\sum_{\substack{s(e)=v \\ r(e) \notin H}} s_{e} s_{e}^{*} .
$$

Definition 2.7. If $H$ is a saturated hereditary subset of $G$ and $S \subseteq B_{H}$, then we define a graph $G_{(H, S)}$ as follows:

$$
\begin{aligned}
& G_{(H, S)}^{0}:=\left(G^{0} \backslash H\right) \cup\left\{v^{\prime}: v \in B_{H} \backslash S\right\}, \\
& G_{(H, S)}^{1}:=\left\{e \in G^{1}: r(e) \notin H\right\} \cup\left\{e^{\prime}: r(e) \in B_{H} \backslash S\right\},
\end{aligned}
$$

and we extend $r$ and $s$ to $G_{(H, S)}^{1}$ by $r\left(e^{\prime}\right)=r(e)^{\prime}$ and $s\left(e^{\prime}\right)=s(e)$. It follows from [1, Corollary 3.5] that $C^{*}(G) / I_{(H, S)} \cong C^{*}\left(G_{(H, S)}\right)$.

\section{Stability of GRAPH $C^{*}$-ALgebras}

This section is devoted to proving Theorem 3.2, which is a generalization of [8] Theorem 2.14].

Definition 3.1. If $v$ is a vertex in a graph $G$ we define $L(v):=\left\{w \in G^{0}: w \geq v\right\}$. We say that $v$ is left infinite if $L(v)$ contains infinitely many elements, and we say that $v$ is left finite if $L(v)$ contains finitely many elements.

Theorem 3.2. If $G$ is a graph, then the following are equivalent.

(a) $C^{*}(G)$ is stable.

(b) $C^{*}(G)$ has no nonzero unital quotients and no tracial states.

(c) Every vertex in $G$ that is on a loop is left infinite and $T(G)=\emptyset$.

(d) Every vertex in $G$ that is on a loop is left infinite and $G$ has no nonzero bounded graph traces.

(e) For every $v \in G^{0}$ and every finite set $F \subseteq G^{0}$, there exists a finite set $W \subseteq G^{0}$ with $W \cap F=\emptyset$ and $p_{v} \lesssim \sum_{w \in W} p_{w}$. 
(f) For every finite set $V \subseteq G^{0}$, there exists a finite set $W \subseteq G^{0}$ with $V \cap W=\emptyset$ and $\sum_{v \in V} p_{v} \lesssim \sum_{w \in W} p_{w}$.

Corollary 3.3. If $G$ is a graph and every vertex of $G$ is left infinite, then $C^{*}(G)$ is stable. If $G$ has no sources and $C^{*}(G)$ is stable, then every vertex of $G$ is left infinite.

Proof. Suppose every vertex of $G$ is left infinite. If $v \in G^{0}$ and $F \subseteq G^{0}$ is a finite set, then we may choose an element $w \in G^{0}$ such that $w \notin F$ and $w \geq v$. But then $p_{v} \lesssim p_{w}$ and by Theorem $3.2(\mathrm{e}), C^{*}(G)$ is stable.

If $G$ has no sources, then for every $v \in G^{0}$ there exists a sequence of edges $e_{1} e_{2} e_{3} \ldots$ with $r\left(e_{i+1}\right)=s\left(e_{i}\right)$ and $r\left(e_{1}\right)=v$. If the elements of $\left\{s\left(e_{i}\right)\right\}_{i=1}^{\infty}$ are distinct, then $v$ is left infinite. If the elements of $\left\{s\left(e_{i}\right)\right\}_{i=1}^{\infty}$ are not distinct, then there exists a loop that can reach $v$. If $C^{*}(G)$ is stable, then by Theorem 3.2(c) all vertices on loops are left infinite. Hence $v$ is also left infinite.

We cannot remove the condition of no sources in the converse of the above corollary. If $G$ is the graph

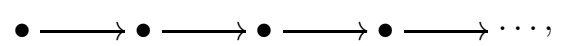

then no vertex of $G$ is left infinite, but $C^{*}(G) \cong \mathcal{K}$ is stable.

Remark 3.4. The equivalence of Conditions (a), (b), and (f) in Theorem 3.2 was established for locally finite graphs in [8, Theorem 2.14]. We mention that Condition (c) of Theorem 3.2 is often easier to verify than Condition (b). This is because graph traces are typically easier to deal with than tracial states, and it is often easy to deduce whether $T(G)$ is empty simply by looking at $G$. Furthermore, we point out that the tracial states of $C^{*}(G)$ and the graph traces on $G$ of norm one are not generally in one-to-one correspondence (see [12, §3]).

Remark 3.5. We see from Theorem 3.2 that a graph $C^{*}$-algebra is stable if and only if it has no nonzero unital quotients and no tracial states. It is always the case that any stable $C^{*}$-algebra will have no nonzero unital quotients and no tracial states, but in general the converse does not hold. (Interestingly, it is shown in [9. Proposition 5.1] that the converse will hold if certain full hereditary subalgebras of the $C^{*}$-algebra satisfy a particular property.)

Lemma 3.6. Let $A$ be a $C^{*}$-algebra with an increasing countable approximate unit $\left\{p_{n}\right\}_{n=1}^{\infty}$ consisting of projections. Then the following are equivalent.

(i) A is stable.

(ii) For every projection $p \in A$, there exists a projection $q \in A$ such that $p \sim q$ and $p \perp q$.

(iii) For all $n \in \mathbb{N}$, there exists $m>n$ such that $p_{n} \lesssim p_{m}-p_{n}$.

Proof. The equivalence of (i) and (ii) is shown in [9, Theorem 3.3]. The equivalence of (ii) and (iii) is shown in [8, Lemma 2.1].

Lemma 3.7. If $G$ is a graph and $g: G^{0} \rightarrow \mathbb{R}^{+}$is a graph trace on $G$, then

$$
H:=\left\{v \in G^{0}: g(v)=0\right\}
$$

is a saturated hereditary subset. 
Proof. If $e \in G^{1}$, then $g(s(e)) \geq g(r(e))$. Thus $s(e) \in H$ implies that $r(e) \in H$, and $H$ is hereditary. If $v \in G^{0}$ is not a singular vertex and $\left\{r(e): e \in G^{1}\right.$ and $s(e)=$ $v\} \subseteq H$, then $g(v)=\sum_{s(e)=v} g(r(e))=0$. So $v \in H$, and $H$ is saturated.

Lemma 3.8. Let $G$ be a graph, let $H$ be a saturated hereditary subset of $G^{0}$, and let $\pi: C^{*}(G) \rightarrow C^{*}(G) / I_{(H, \emptyset)}$ be the projection map. If $p$ is a projection in $C^{*}(G)$, $W \subseteq G^{0} \backslash H$ is a finite set, and $\pi(p) \lesssim \sum_{w \in W} \pi\left(p_{w}\right)$ in $C^{*}(G) / I_{(H, \emptyset)}$, then there exists a finite set $X \subseteq H$ such that $p \lesssim \sum_{w \in W} p_{w}+\sum_{x \in X} p_{x}$ in $C^{*}(G)$.

Proof. Write $H=\left\{v_{1}, v_{2}, \ldots\right\}$. If we let $p_{n}:=\sum_{i=1}^{n} p_{v_{i}}$, then $I_{(H, \emptyset)}$ is generated by $\mathcal{P}=\left\{p_{n}\right\}_{n=1}^{\infty}$ and [8, Lemma 2.6] implies that $p \lesssim \sum_{w \in W} p_{w}+p_{n}$ for some $n$.

Proof of Theorem [3.2. (a) $\Longrightarrow$ (b) : It is shown in [9. Proposition 5.1] that stable $C^{*}$-algebras have no nonzero unital quotients and admit no nonzero traces.

(b) $\Longrightarrow($ c) : We shall first show that every vertex on a loop is left infinite. Let $\alpha$ be a loop in $G$ that is based at $v$. Then $H:=G^{0} \backslash L(s(\alpha))$ is a saturated hereditary subset. By hypothesis $C^{*}(G) / I_{\left(H, B_{H}\right)} \cong G_{\left(H, B_{H}\right)}$ is nonunital, and hence $G_{\left(H, B_{H}\right)}^{0}=G^{0} \backslash H=L(s(\alpha))$ is infinite. Thus $s(\alpha)=v$ is left infinite.

Now we shall show that $T(G)$ is empty by supposing that there exists $g \in T(G)$ and arriving at a contradiction. Let us begin by showing that if $v$ is a vertex on a loop, then $g(v)=0$. From the previous paragraph every vertex on a loop is left infinite. Since

$$
\|g\|=\sum_{w \in G^{0}} g(w) \geq \sum_{w \in L(v)} g(w)
$$

and since $w \geq v$ implies $g(w) \geq g(v)$, the only way that this infinite sum can be finite is if $g(v)=0$. Thus $g$ vanishes on every vertex that is on a loop.

If we now let $H:=\left\{v \in G^{0}: g(v)=0\right\}$, then it follows from Lemma 3.7 that $H$ is a saturated hereditary subset. We define a graph trace $\tilde{g}$ on $G_{(H, \emptyset)}$ by

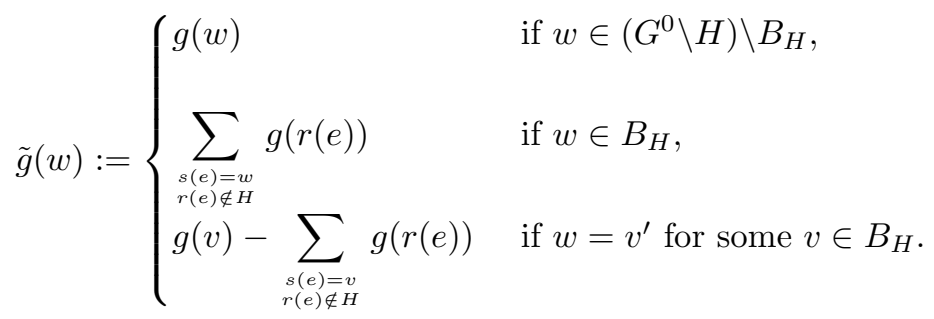

It is straightforward to verify that $\tilde{g}$ is a graph trace on $G_{(H, \emptyset)}$ and that $\|\tilde{g}\|=\|g\|=$ 1. Now it follows from the previous paragraph that there are no loops in $G$ with vertices in $G^{0} \backslash H$. Hence $G_{(H, \emptyset)}$ is a graph with no loops. Therefore [12, §3.3] implies that there exists a tracial state $\tau$ on $C^{*}\left(G_{(H, \emptyset)}\right)$. Since $C^{*}(G) / I_{(H, \emptyset)} \cong C^{*}\left(G_{(H, \emptyset)}\right)$, it follows that $\tau$ lifts to a tracial state on $C^{*}(G)$. But this contradicts the fact that $C^{*}(G)$ has no tracial states.

(c) $\Longrightarrow($ d) : If $g$ was a nonzero bounded graph trace on $G$, then we could normalize to get an element $\frac{1}{\|g\|} \cdot g \in T(G)$.

$(\mathrm{d}) \Longrightarrow(\mathrm{e})$ : Choose a vertex $v \in G^{0}$. Define $H:=\left\{w \in G^{0}: w\right.$ is left infinite $\}$. Then $H$ is a hereditary subset, and we let $\bar{H}$ denote the saturation of $H$. Consider the following two cases:

Case I: $v \in \bar{H}$. Define $H_{0}:=H$ and for each $n \in \mathbb{N}$ set

$$
H_{n+1}:=H_{n} \cup\left\{w \in G^{0}: 0<\left|s^{-1}(w)\right|<\infty \text { and } s(e)=w \text { implies } r(e) \in H_{n}\right\} \text {. }
$$


Then we see that $\bar{H}=\bigcup_{n=0}^{\infty} H_{n}$. We shall prove that the claim holds whenever $v \in \bar{H}$ by induction on $k:=\min \left\{n \in \mathbb{N}: v \in H_{n}\right\}$. In the base case we have $k=0$ and thus $v \in H$. Since every vertex in $H$ is left infinite for every finite set $F \subseteq G^{0}$, there exists $w \in G^{0}$ such that $w \notin F$ and $w \geq v$. But then $p_{v} \lesssim p_{w}$ and the claim holds. Now assume that the claim holds whenever $v$ is in $\bar{H}$ with $\min \{n \in \mathbb{N}: v \in$ $\left.H_{n}\right\}$ strictly less than a fixed $k$. Suppose that $v \in H_{k}$. Then $s^{-1}(v)$ consists of a finite and nonzero number of edges $\left\{e_{1}, \ldots, e_{n}\right\}$ with $r\left(e_{i}\right) \in H_{k-1}$ for all $i$. By the induction hypothesis there exists a finite set $W_{1} \subseteq G^{0}$ such that $W_{1} \cap F=\emptyset$ and $p_{r\left(e_{1}\right)} \lesssim \sum_{w \in W_{1}} p_{w}$. Similarly for each $1<i \leq n$, there exists a finite set $W_{i} \subseteq G^{0}$ that is disjoint from $F \cup W_{1} \cup \ldots \cup W_{i-1}$ and with $p_{r\left(e_{i}\right)} \lesssim \sum_{w \in W_{i}} p_{w}$. Now if we let $x=s_{e_{1}}+\ldots+s_{e_{n}}$ then we see that $x^{*}\left(\sum_{i=1}^{n} s_{e_{i}} s_{e_{i}}^{*}\right) x=\sum_{i=1}^{n} p_{r\left(e_{i}\right)}$, and thus $\sum_{i=1}^{n} s_{e_{i}} s_{e_{i}}^{*} \lesssim \sum_{i=1}^{n} p_{r\left(e_{i}\right)}$. Therefore if we let $W:=W_{1} \cup \ldots \cup W_{n}$ we see that $W \cap F=\emptyset$ and $p_{v}=\sum_{i=1}^{n} s_{e_{i}} s_{e_{i}}^{*} \lesssim \sum_{i=1}^{n} p_{r\left(e_{i}\right)} \lesssim \sum_{w \in W_{1}} p_{w}+\ldots \sum_{w \in W_{n}} p_{w}=$ $\sum_{w \in W} p_{w}$.

CASE II: $v \notin \bar{H}$. Since every vertex on a loop is left infinite, it follows that no vertices of $G^{0} \backslash \bar{H}$ are on loops. Thus $G_{(\bar{H}, \emptyset)}$ contains no loops and [6, Corollary 2.13] implies that $C^{*}\left(G_{(\bar{H}, \emptyset)}\right)$ is an AF-algebra. Furthermore, there are no tracial states on $C^{*}\left(G_{(\bar{H}, \emptyset)}\right) \cong C^{*}(G) / I_{(\bar{H}, \emptyset)}$ since any tracial state would lift to a tracial state on $C^{*}(G)$ and thus induce a graph trace of norm one on $G$. Since $C^{*}\left(G_{(\bar{H}, \emptyset)}\right)$ is an AF-algebra with no tracial states, it follows from [3 Theorem 4.10] that it is stable.

If we list the vertices of $G^{0} \backslash \bar{H}$ as $\left\{w_{1}, w_{2}, \ldots\right\}$ with $w_{1}=v$, then the elements $p_{n}:=\sum_{i=1}^{n} \pi\left(p_{w_{i}}\right)$ form an increasing approximate unit for $C^{*}\left(G_{(\bar{H}, \emptyset)}\right)$ consisting of projections. If $F \subseteq G^{0}$ is a finite set, let $n=\max \left\{i \in \mathbb{N}: w_{i} \in F\right\}$. Since $C^{*}\left(G_{(H, \emptyset)}\right)$ is stable, Lemma 3.6)(c) implies that there exists $m>n$ such that $p_{n} \lesssim p_{m}-p_{n}$. But if we let $W_{0}:=\left\{w_{n+1}, \ldots, w_{m}\right\}$, then $W_{0} \cap F=\emptyset$ and $\pi\left(p_{v}\right) \lesssim p_{n} \lesssim p_{m}-p_{n}=\sum_{w \in W_{0}} \pi\left(p_{w}\right)$. It then follows from Lemma 3.8 that there exists a finite set $X \subseteq \bar{H}$ for which $p_{v} \lesssim \sum_{w \in W} p_{w}+\sum_{x \in X} p_{x}$ in $C^{*}(G)$. Now since $X \subseteq \bar{H}$ we see from Case I above that if $X=\left\{x_{1}, \ldots, x_{n}\right\}$, then for each $i$ we may choose $W_{i}$ such that $W_{i}$ is disjoint from $F \cup W_{0} \cup \ldots W_{i-1}$ and $p_{x_{i}} \lesssim \sum_{w \in W_{i}} p_{w}$. If we let $W:=W_{0} \cup \ldots \cup W_{n}$, then $W \cap F=\emptyset$ and $p_{v} \lesssim \sum_{w \in W_{0}} p_{w}+\sum_{x \in X} p_{x} \lesssim$ $\sum_{w \in W_{0}} p_{w}+\ldots+\sum_{w \in W_{n}} p_{w}=\sum_{w \in W} p_{w}$.

(e) $\Longrightarrow$ (f) : List the elements of $V$ as $V=\left\{v_{1}, \ldots, v_{n}\right\}$. Choose $W_{1}$ such that $W_{1} \cap V=\emptyset$ and $p_{v_{1}} \lesssim \sum_{w \in W_{1}} p_{w}$. Having chosen $W_{k}$ we may choose $W_{k+1}$ so that $W_{k+1}$ is disjoint from $V \cup W_{1} \cup \ldots \cup W_{k}$ and $p_{v_{k}} \lesssim \sum_{w \in W_{k}} p_{w}$. We continue in this fashion until we produce $n$ sets $W_{1}, \ldots, W_{n}$ with these properties. If we let $W:=W_{1} \cup \ldots \cup W_{n}$, then $V \cap W=\emptyset$ and $\sum_{v \in V} p_{v} \lesssim \sum_{w \in W_{1}} p_{w}+\ldots+\sum_{w \in W_{n}} p_{w}=$ $\sum_{w \in W} p_{w}$.

(f) $\Longrightarrow$ (a) : List the vertices of $G$ as $G^{0}:=\left\{v_{1}, v_{2}, \ldots\right\}$. For each $n \in \mathbb{N}$ we define $p_{n}:=\sum_{i=1}^{n} p_{v_{i}}$. Then $\left\{p_{n}\right\}_{n=1}^{\infty}$ is an increasing approximate unit consisting of projections, and by Lemma 3.6 it suffices to prove that for all $n \in N$ there exists $m>n$ such that $p_{n} \lesssim p_{m}-p_{n}$.

Let $n \in N$, and define $V:=\left\{v_{1}, \ldots, v_{n}\right\}$. By hypothesis there exists a finite set $W \subseteq G^{0}$ such that $V \cap W=\emptyset$ and $\sum_{v \in V} p_{v} \lesssim \sum_{w \in W} p_{w}$. Let $m:=\max \{k \in$ $\left.\mathbb{N}: v_{k} \in W\right\}$. Since $V \cap W=\emptyset$ we see that $\sum_{w \in W} p_{v} \leq p_{m}-p_{n}$. Thus $p_{n}=$ $\sum_{v \in V} p_{v} \lesssim \sum_{v \in W} p_{v} \leq p_{m}-p_{n}$. 


\section{The Stabilization of A GRAPH $C^{*}$-ALGEBRA}

Definition 4.1. If $G$ is a graph and $v \in G^{0}$ is a vertex, then by adding a head to $v$ we mean attaching a graph of the form

$$
\cdots \stackrel{e_{4}}{\longrightarrow} v_{3} \stackrel{e_{3}}{\longrightarrow} v_{2} \stackrel{e_{2}}{\longrightarrow} v_{1} \stackrel{e_{1}}{\longrightarrow} v .
$$

Thus we create a new graph $F$ from $G$ by defining $F^{0}:=G^{0} \cup\left\{v_{1}, v_{2}, \ldots\right\}, F^{1}:=$ $G^{1} \cup\left\{e_{1}, e_{2}, \ldots\right\}$, and extend $r$ and $s$ to $F^{1}$ by $r\left(e_{i}\right)=v_{i-1}$ and $s\left(e_{i}\right)=v_{i}$.

The terminology "adding a head" is meant to complement the terminology for the analogous concept of "adding a tail" introduced in [2] (1.2)].

Theorem 4.2. If $G$ is a graph, let $\tilde{G}$ be the graph obtained by adding a head to each vertex of $G$. Then $C^{*}(\tilde{G})$ is the stabilization of $C^{*}(G)$; that is,

$$
C^{*}(\tilde{G}) \cong C^{*}(G) \otimes \mathcal{K} .
$$

Proof. Following the proof of [2, Lemma 1.2] one can show that $C^{*}(G)$ is naturally isomorphic to a full corner of $C^{*}(\tilde{G})$. Consequently, $C^{*}(G)$ is Morita equivalent to $C^{*}(\tilde{G})$, and since Corollary 3.3 implies that $C^{*}(\tilde{G})$ is stable we have $C^{*}(G) \otimes \mathcal{K} \cong$ $C^{*}(\tilde{G}) \otimes \mathcal{K} \cong C^{*}(\tilde{G})$.

Corollary 4.3. The class of graph $C^{*}$-algebras is closed under stabilization.

Example 4.4. If $G$ is the graph

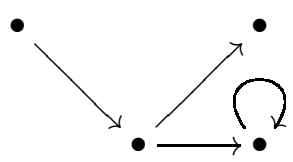

then $\tilde{G}$ is the graph

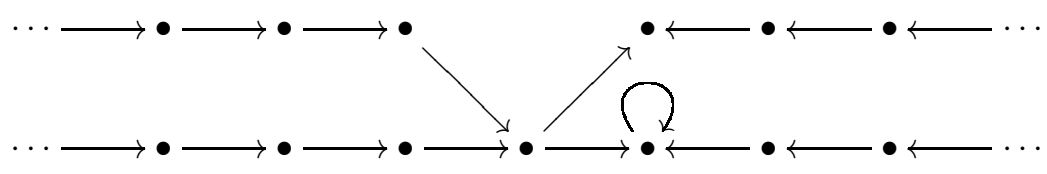

and $C^{*}(\tilde{G}) \cong C^{*}(G) \otimes \mathcal{K}$.

Example 4.5. If $G$ is the following graph with one vertex and infinitely many edges, then $C^{*}(G) \cong \mathcal{O}_{\infty}$

$$
\bullet \infty
$$

and $\tilde{G}$ is the graph

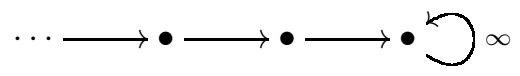

so that $C^{*}(\tilde{G}) \cong \mathcal{O}_{\infty} \otimes \mathcal{K}$.

Remark 4.6. To obtain the stabilization it is often unnecessary to add a head to every vertex in $G$. It suffices to add enough heads to make all vertices left infinite. For example, one could choose to add heads only at the left finite vertices of $G$. 


\section{REFERENCES}

[1] T. Bates, J. H. Hong, I. Raeburn, and W. Szymański, The ideal structure of the $C^{*}$-algebras of infinite graphs, Illinois J. Math. 46 (2002), 1159-1176.

[2] T. Bates, D. Pask, I. Raeburn, and W. Szymański, The $C^{*}$-algebras of row-finite graphs, New York J. Math. 6 (2000), 307-324 (electronic). MR 2001k:46084

[3] B. Blackadar, Traces on simple AF $C^{*}$-algebras, J. Funct. Anal. 38 (1980), 156-168. MR 82a:46062

[4] J. Cuntz, Dimension functions on simple $C^{*}$-algebras, Math. Ann. 233 (1978), 145-153. MR 57:7191

[5] J. Cuntz and W. Krieger, A class of $C^{*}$-algebras and topological Markov chains, Invent. Math. 56 (1980), 251-268. MR 82f:46073a

[6] D. Drinen and M. Tomforde, The $C^{*}$-algebras of arbitrary graphs, preprint (2000).

[7] N. Fowler, M. Laca, and I. Raeburn, The $C^{*}$-algebras of infinite graphs, Proc. Amer. Math. Soc. 128 (2000), 2319-2327. MR 2000k:46079

[8] J. Hjelmborg, Purely infinite and stable $C^{*}$-algebras of graphs and dynamical systems, Ergodic Theory Dynam. Systems 21 (2001), 1789-1808. MR 2002h:46112

[9] J. Hjelmborg and M. Rørdam, On stability of $C^{*}$-algebras, J. Funct. Anal. 155 (1998), 153170. MR 99g:46079

[10] A. Kumjian, D. Pask, and I. Raeburn, Cuntz-Krieger algebras of directed graphs, Pacific J. Math. 184 (1998), 161-174. MR 99i:46049

[11] A. Kumjian, D. Pask, I. Raeburn, and J. Renault, Graphs, groupoids, and Cuntz-Krieger algebras, J. Funct. Anal. 144 (1997), 505-541. MR 98g:46083

[12] M. Tomforde, The ordered $K_{0}$-group of a graph $C^{*}$-algebra, C. R. Math. Acad. Sci. Soc. R. Can. 25 (2003), 19-25. MR 2003m:46104

[13] Y. Watatani, Graph theory for $C^{*}$-algebras, in Operator Algebras and Their Applications (R. V. Kadison, ed.), Proc. Sympos. Pure Math., vol. 38, part 1, Amer. Math. Soc., Providence, RI, 1982, pp. 195-197. MR 84a:46124

Department of Mathematics, Dartmouth College, Hanover, New Hampshire 037553551

Current address: Department of Mathematics, University of Iowa, Iowa City, Iowa 52242

E-mail address: tomforde@math.uiowa.edu 